\title{
An Improved Sensitivity Method for the Simultaneous Identification of Unknown Parameters and External Loads of Nonlinear Structures
}

\author{
Chongwen Wang $(i)$ and Chengbin Du \\ Department of Engineering Mechanics, Hohai University, Nanjing 211100, China \\ Correspondence should be addressed to Chengbin Du; cbdu@hhu.edu.cn
}

Received 8 June 2018; Revised 27 September 2018; Accepted 15 October 2018; Published 7 November 2018

Academic Editor: Matthew J. Whelan

Copyright (c) 2018 Chongwen Wang and Chengbin Du. This is an open access article distributed under the Creative Commons Attribution License, which permits unrestricted use, distribution, and reproduction in any medium, provided the original work is properly cited.

\begin{abstract}
Because structures may be subject to unknown loads and may simultaneously involve unknown parameters and because simple load identification or parameter identification algorithms cannot be applied under such conditions, it is necessary to seek algorithms that can simultaneously identify unknown parameters and external loads of structures. The sensitivity method is one of them, and this paper extends this method to nonlinear structures. In addition, the key issues associated with the sensitivity method are systematically studied, and suggestions for improvement are put forward, including the use of the difference method instead of the derivative method to calculate the sensitivity, the use of a fixed regularization parameter instead of the traditional regularization parameter calculation methods, and measures for guarantee of iterative convergence. The improved sensitivity method is applied to two types of nonlinear structures, and the effects of the regularization parameter, distribution of measured points, response types, noise levels, and the magnitude of the perturbation on the identified results are discussed.
\end{abstract}

\section{Introduction}

Structural health monitoring [1] is widely used to assess whether a structure is damaged and to estimate damage location and degree. Therefore, structural health monitoring often relies on damage identification algorithms [2]. In general, the quantification of damage is often characterized by the changes in structural parameters before and after the damage occurs; thus, many damage identification algorithms [3] first identify structural parameters and then determine the damage degree by comparing the identified value and design value.

The inverse problem in structural dynamics involves two main types: load identification [4] and parameter identification [5], where the identification of one often requires the other to be known. For engineering structures, because of the limitations imposed by technical conditions, dynamic loads are often very difficult to measure directly. Moreover, many parameters cannot be accurately obtained through simple tests, such as damping parameters, particularly when the structure is damaged. Therefore, unknown parameters and unknown loads often exist in a structure at the same time.

Some early structural parameter identification theories were developed without considering input information. For example, Ibrahim [6] directly used the free vibration response to identify structural parameters, and Toki et al. [7] assumed that the end of the response under earthquake excitation is free vibration. In addition, in the field of modal parameter identification [8], it is usually assumed that the input follows a Gaussian distribution, such as in the time series analysis [9], natural excitation technique (NExT) [10], and stochastic subspace identification (SSI) [11].

For the abovementioned reasons, algorithms for simultaneously identifying structural parameters and loads have been developed. Maruyama and Hoshiya et al. [12, 13] described a moving load by several parameters, such as the moving speed and the load size, and introduced these parameters into the structural state equation; subsequently, the extended Kalman filter (EKF) was used for the identification 
of loads and parameters. Yang et al. [14] proposed a recursive least-squares estimation with unknown input (RLSE-UI) algorithm to identify unknown parameters and input. Later, Yang and Huang [15] proposed a refined algorithm referred to as adaptive sequential nonlinear leastsquares estimation with unknown input (ASNLSE-UI) for real-time identification of damage and input, and a series of experiments are performed to verify the applicability of this algorithm [16]. Zhu and Law [17] proposed a two-step inversion method to identify the moving loads and damage of an Euler-Bernoulli beam. In that work, the Dirac function was adopted to simulate cracks in a beam. Lu and Law [18] proposed a composite algorithm based on response sensitivity with respect to external loads and unknown parameters, referred to as the sensitivity method in this paper. Zhang et al. [19, 20] identified loads and damage based on the difference in responses between the undamaged and damaged structure via the virtual deformation method (VDM). Xu et al. [21] proposed an identification algorithm referred to as weighted adaptive iterative least-squares estimation with incomplete measured excitations (WAILSEIME) to identify unknown parameters and unknown external loads. A learning coefficient and a weighted positive definite matrix were introduced to improve the convergence speed and accuracy.

Based on the basic idea of the sensitivity method proposed in [18], this paper extends this method to nonlinear structures and describes studies of three key issues associated with this method, including the calculation of the sensitivity, the determination of the regularization parameter, and iterative convergence. The improved sensitivity method is applied to two types of nonlinear structures. In addition, the effects of the regularization parameter, distribution of measured points, response types, noise levels, and the magnitude of the perturbation on the identified results are discussed.

\section{The Basic Idea of the Sensitivity Method for a Nonlinear Structure}

2.1. Calculation of the Sensitivity. For a multi-degree-offreedom nonlinear structure, the vibration equation can be expressed as

$$
\mathbf{M} \ddot{\mathbf{y}}(t)+\mathbf{F}[\dot{\mathbf{y}}(t), \mathbf{y}(t), \mathbf{p}]=\mathbf{B}_{f} \mathbf{f}(t),
$$

where $\mathbf{M}$ is the mass matrix; $\ddot{\mathbf{y}}(t), \dot{\mathbf{y}}(t)$, and $\mathbf{y}(t)$ are the acceleration, velocity, and displacement vectors, respectively; $\mathbf{p}$ is the vector of unknown parameters; $\mathbf{F}$ is a nonlinear function of the displacement, velocity, and unknown structural parameters; $\mathbf{f}(t)$ is the external load vector; and $\mathbf{B}_{f}$ is the allocation matrix associated with $\mathbf{f}(t)$.

The $i$-th external load $f^{i}(t)$ can be orthogonally expanded by the Legendre polynomials:

$$
f^{i}(t)=\sum_{m=1}^{N_{m}} c_{m}^{i} L_{m}(t), \quad\left(i=1,2, \ldots, N_{f}\right),
$$

where $c_{m}^{i}$ is the $m$-th orthogonal expansion coefficient of $f^{i}(t) ; L_{m}(t)$ is the $m$-th Legendre polynomial; $N_{m}$ is the number of items of $f^{i}(t)$ expanded by the Legendre polynomials; and $N_{f}$ is the number of external loads. The Legendre polynomials can be written as

$$
\begin{aligned}
L_{1}(t) & =1, \\
L_{2}(t) & =\frac{2 t}{T}-1, \\
L_{m+1}(t) & =\frac{2 m-1}{m}\left(\frac{2 t}{T}-1\right) L_{m}(t)-\frac{m-1}{m} L_{m-1}(t), \\
& \left(m=2,3, \ldots, N_{m}-1\right),
\end{aligned}
$$

where $T$ indicates that the defined interval of the Legendre polynomials is $[0, T]$.

Substituting (2) into (1) yields

$$
\begin{aligned}
& \mathbf{M y}(t)+\mathbf{F}[\dot{\mathbf{y}}(t), \mathbf{y}(t), \mathbf{p}] \\
& \quad=\mathbf{B}_{f}\left[\sum_{m=1}^{N_{m}} c_{m}^{1} L_{m}(t) \sum_{m=1}^{N_{m}} c_{m}^{2} L_{m}(t) \cdots \sum_{m=1}^{N_{m}} c_{m}^{N_{f}} L_{m}(t)\right]^{\prime},
\end{aligned}
$$

where the superscript " ()$^{\prime \prime}$ " represents matrix transposition.

Because the mass is generally easy to measure and there is no significant change in mass when a structure is damaged, the following calculation assumes that the mass is known. Meanwhile, suppose that the structure involves $N_{l}$ unknown parameters, denoted by $p_{l}\left(l=1,2, \ldots, N_{l}\right)$. By calculating the partial derivative of (4) with respect to $c_{m}^{i}$ and $p_{l}$, respectively, the following two equations can be obtained:

$$
\begin{aligned}
& \mathbf{M} \frac{\partial \ddot{\mathbf{y}}(t)}{\partial c_{m}^{i}}+\frac{\partial \mathbf{F}}{\partial \dot{\mathbf{y}}(t)} \frac{\partial \dot{\mathbf{y}}(t)}{\partial c_{m}^{i}}+\frac{\partial \mathbf{F}}{\partial \mathbf{y}(t)} \frac{\partial \mathbf{y}(t)}{\partial c_{m}^{i}}=\mathbf{B}_{f}\left[\begin{array}{lll}
\mathbf{0} & L_{m}(t) & \mathbf{0}
\end{array}\right]^{\prime}, \\
& \left(m=1,2, \ldots, N_{m}, i=1,2, \ldots, N_{f}\right),
\end{aligned}
$$

$$
\begin{array}{r}
\mathbf{M} \frac{\partial \ddot{\mathbf{y}}(t)}{\partial p_{l}}+\frac{\partial \mathbf{F}}{\partial \dot{\mathbf{y}}(t)} \frac{\partial \dot{\mathbf{y}}(t)}{\partial p_{l}}+\frac{\partial \mathbf{F}}{\partial \mathbf{y}(t)} \frac{\partial \mathbf{y}(t)}{\partial p_{l}}=-\frac{\partial \mathbf{F}}{\partial \mathbf{p}} \frac{\partial \mathbf{p}}{\partial p_{l}} \\
\left(l=1,2, \ldots, N_{l}\right)
\end{array}
$$

After initial values are assumed for the unknown parameters and orthogonal expansion coefficients of loads, $\ddot{\mathbf{y}}(t), \dot{\mathbf{y}}(t)$, and $\mathbf{y}(t)$ can be obtained by solving (4); then, the sensitivity of the response with respect to orthogonal expansion coefficients of loads and unknown parameters can be obtained by solving (5) and (6), respectively.

\subsection{The Establishment of and Solution to the Governing} Equation. First, the response is submitted to Taylor expansion at the initial values, and only the first-order items are retained. Taking the acceleration as an example, the following equation can be obtained:

$$
\ddot{\mathbf{y}}_{a}=\mathbf{L}\left(\ddot{\mathbf{y}}_{c}\right)+\sum_{i=1}^{N_{f}} \sum_{m=1}^{N_{m}} \frac{\partial \ddot{\mathbf{y}}_{c}(t)}{\partial c_{m}^{i}} \Delta c_{m}^{i}+\sum_{l=1}^{N_{l}} \frac{\partial \ddot{\mathbf{y}}_{c}(t)}{\partial p_{l}} \Delta p_{l},
$$

where $\ddot{\mathbf{y}}_{a}$ is the measured acceleration treated as a known quantity; $\ddot{\mathbf{y}}_{c}$ is the acceleration calculated by (4); L is 
a mapping matrix selecting the response from $\ddot{\mathbf{y}}_{c}$ with the same degrees of freedom as $\ddot{\mathbf{y}}_{a}$; and $\Delta c_{m}^{i}\left(m=1,2, \ldots, N_{m}\right.$, $\left.i=1,2, \ldots, N_{f}\right)$ and $\Delta p_{l}\left(l=1,2, \ldots, N_{l}\right)$ are the perturbations of unknown parameters and orthogonal expansion coefficients of loads, respectively.

Thus, the governing equation for solving the unknown parameters and loads can be established:

$$
\ddot{\mathbf{y}}_{a}-\mathbf{L}\left(\ddot{\mathbf{y}}_{c}\right)=\left[\mathbf{S}_{f} \mathbf{S}_{p}\right]\left\{\begin{array}{c}
\Delta \mathbf{c} \\
\Delta \mathbf{p}
\end{array}\right\},
$$

where $\quad \mathbf{S}_{f}=\left[\left(\partial \ddot{\mathbf{y}}_{c}(t) / \partial c_{1}^{1}\right)\left(\partial \ddot{\mathbf{y}}_{c}(t) / \partial c_{2}^{1}\right) \cdots\left(\partial \ddot{\mathbf{y}}_{c}(t) / \partial c_{N_{m}}^{1}\right)\right.$ $\left.\left(\partial \ddot{\mathbf{y}}_{c}(t) / \partial c_{1}^{2}\right) \cdots\left(\partial \ddot{\mathbf{y}}_{c}(t) / \partial c_{N_{m}}^{N_{f}}\right)\right]$ and $\mathbf{S}_{p}=\left[\left(\partial \ddot{\mathbf{y}}_{c}(t) / \partial p_{1}\right)\right.$ $\left.\left(\partial \ddot{\mathbf{y}}_{c}(t) / \partial p_{2}\right) \cdots\left(\partial \ddot{\mathbf{y}}_{c}(t) / p_{N_{l}}\right)\right]$ are the sensitivity matrices, $\Delta \mathbf{c}=\left[\Delta c_{1}^{1} \Delta c_{2}^{1} \cdots \Delta c_{N_{m}}^{1} \Delta c_{1}^{2} \cdots \Delta c_{N_{m}}^{N_{f}}\right]^{\prime}, \quad$ and $\quad \Delta \mathbf{p}=\left[\Delta p_{1}\right.$ $\left.\Delta p_{2} \cdots \Delta p_{N_{l}}\right]^{\prime}$.

The above equation can be simplified as

$$
\Delta \ddot{\mathbf{y}}=\mathbf{S}(\Delta \boldsymbol{\alpha}) \text {. }
$$

Subsequently, (9) can be solved by the Tikhonov regularization method [22]:

$$
\Delta \boldsymbol{\alpha}=\left(\mathbf{S}^{\prime} \mathbf{S}+\lambda \mathbf{E}\right)^{-1} \mathbf{S}^{\prime}(\Delta \ddot{\mathbf{y}}),
$$

where $\lambda>0$, which is the regularization parameter, and $E$ is an identity matrix. The regularization parameter can be calculated by the L-curve criterion [23] or the generalized cross-validation (GCV) criterion [24].

Finally, the calculated $\Delta \boldsymbol{\alpha}$ is added to the initial values of the unknown parameters and orthogonal expansion coefficients of loads as the identified values at this iteration step and the initial values at the next iteration step, and the next iteration step is performed until the identified results meet the convergence condition tolerance:

$$
\left|\frac{\left(\alpha_{i}^{k}-\alpha_{i}^{k-1}\right)}{\alpha_{i}^{k}}\right|<\text { tolerance, } \quad(i=1,2, \ldots),
$$

where $\alpha_{i}^{k}$ and $\alpha_{i}^{k-1}$ are the identified values of a certain unknown quantity $\alpha_{i}$ at the $k$-th and $(k-1)$-th iteration steps, respectively.

\section{Key Issues Associated with the Sensitivity Method}

Even if the sensitivity method is extended to nonlinear structures, the governing equation is still very simple, making this method highly suitable for engineering applications. However, certain key issues still deserve further study.

Based on the governing equation of the sensitivity method, it can be observed that the most important step is naturally the calculation of the sensitivity. From the perspective of solving the governing equation, the key issue is the selection of the regularization method and determination of the regularization parameter. In addition, because the sensitivity method itself is an iterative algorithm, it is necessary to study the iterative convergence.

3.1. Calculation of the Sensitivity. The derivative method is used to calculate sensitivity in [18], but the derivative method is not always suitable. For example, for a singledegree-of-freedom Bouc-Wen model structure, the vibration equation can be expressed as

$$
m \ddot{y}(t)+c \dot{y}(t)+k z(t)=f(t),
$$

where $m, c$, and $k$ are the structural mass, damping, and stiffness, respectively; $\ddot{y}(t)$ and $\dot{y}(t)$ are the acceleration and velocity, respectively; $z(t)$ is the structural hysteretic displacement and $\dot{z}(t)=\dot{y}(t)-\beta|\dot{y}(t) \| z(t)|^{n-1} z(t)-\gamma \dot{y}(t)$ $|z(t)|^{n}$; and $\beta, \gamma$, and $n$ are the Bouc-Wen model parameters.

Because the vibration equation of the Bouc-Wen model structure contains absolute value terms, in calculating the derivative of (12) with respect to unknown parameters and orthogonal expansion coefficients of loads, it is necessary to judge the positive and negative properties of the absolute terms, which will bring great inconvenience.

Therefore, to make the sensitivity method more general, the sensitivity is calculated using the difference method in this paper. First, the initial values are assumed for the unknown quantities in (4), and the response is calculated; then, each unknown quantity is allowed to have a perturbation at the initial value, and the response is calculated again; finally, the difference between the two responses is treated as the sensitivity of the response with respect to this unknown quantity.

\subsection{Selection of the Regularization Method and Determination} of the Regularization Parameter. Because of the inevitable illcondition of the matrix, many inverse problems are illposed. In the field of dynamic load identification, the most common way to solve ill-posed problems is via regularization methods, such as the Tikhonov regularization method or truncated singular value decomposition (TSVD) method [25]. However, regardless of which regularization method is used, the most important step is the determination of the regularization parameter; in this regard, the most common methods are the GCV criterion and L-curve criterion, as previously mentioned.

The fundamental purpose of introducing regularization is to suppress the effect of the noise contained in the response. In the sensitivity method, as the iteration proceeds, the identified values of unknown quantities gradually approach the true values; meanwhile, the calculated response gradually approaches the measured response, which causes $\Delta \ddot{\mathbf{y}}$ in (9) to contain less useful information, and noise gradually becomes a major component, which is different from the conventional load identification problem. A detailed study of the regularization parameter is performed in the examples herein provided.

3.3. Iterative Convergence. Under normal circumstances, because there is no prior knowledge of certain structural parameters and external loads, the assumed initial values may include large errors compared with the true values, and such large errors may lead to significant changes in structural parameters within initial few iteration steps, which may cause the structural parameters to lose physical meaning. 
To solve this problem, it is necessary to introduce a constraint on the identified parameters, that is, to determine a constraint interval for the identified value of $p_{l}$ :

$$
p_{l}^{\text {low }}<p_{l}<p_{l}^{\text {up }}, \quad\left(l=1,2, \ldots, N_{l}\right),
$$

where $p_{l}^{\text {up }}$ and $p_{l}^{\text {low }}$ are the upper and lower bounds of the identified value of $p_{l}$, respectively. When the identified value of $p_{l}$ in a certain iteration step exceeds the constraint interval, this parameter is assigned the value identified in the previous iteration step.

However, the constraint method cannot be applied to the orthogonal expansion coefficients of loads. In addition, for some structural parameters, such as damping and nonlinear parameters, it is difficult to define a relatively small constraint interval, causing the constraint method to lose some significance. The problem of iterative convergence is discussed in the examples herein provided.

\section{Evaluation of the Identified Results}

Because the measured response in practical engineering necessarily contains random noise, the presence of noise will inevitably affect the identified results; that is, even if the response contains the same level of noise, the identified results are definitely not the same. Therefore, a single calculation cannot accurately reflect the stability and accuracy of a method.

Therefore, this paper first incorporates many sets of noise with the same level but different time histories into the response; then, all sets of the response are then used to identify the unknown parameters and loads; finally, the following equation is used to define the identified error of $p_{l}$

$$
\operatorname{err}_{p_{l}}=\frac{1}{N_{c}} \sum_{i=1}^{N_{c}}\left|\frac{p_{l}^{\text {iden }}-p_{l}^{\text {true }}}{p_{l}^{\text {true }}}\right|, \quad\left(l=1,2, \ldots, N_{l}\right),
$$

where ${ }^{i} p_{l}^{\text {iden }}$ and $p_{l}^{\text {true }}$ are, respectively, the identified value of the $i$-th calculation and the true value of $p_{l}$, and $N_{c}$ is the calculation times.

Similarly, the identified error of $f_{j}$ is defined by

$$
\operatorname{err}_{f_{j}}=\frac{1}{N_{c}} \sum_{i=1}^{N_{c}} \frac{\left\|i f_{j}^{\text {iden }}-f_{j}^{\text {true }}\right\|_{2}}{\left\|f_{j}^{\text {true }}\right\|_{2}}, \quad\left(j=1,2, \ldots, N_{f}\right) \text {, }
$$

where ${ }^{i} f_{j}^{\text {iden }}$ and $f_{j}^{\text {true }}$ are the identified value of the $i$-th calculation and the true value of $f_{j}$, respectively, and $\|\cdot\|_{2}$ represents the 2 norms of a vector.

\section{Nonlinear Model Examples}

5.1. A Hard-Spring Model Structure. In this section, a tenstorey shear frame structure is used as the calculation model, as shown in Figure 1, and the numbers in Figure 1 are the number of degrees of freedom.

By assembling the equilibrium equation at each degree of freedom, the vibration equation of this hard-spring model structure can be expressed as

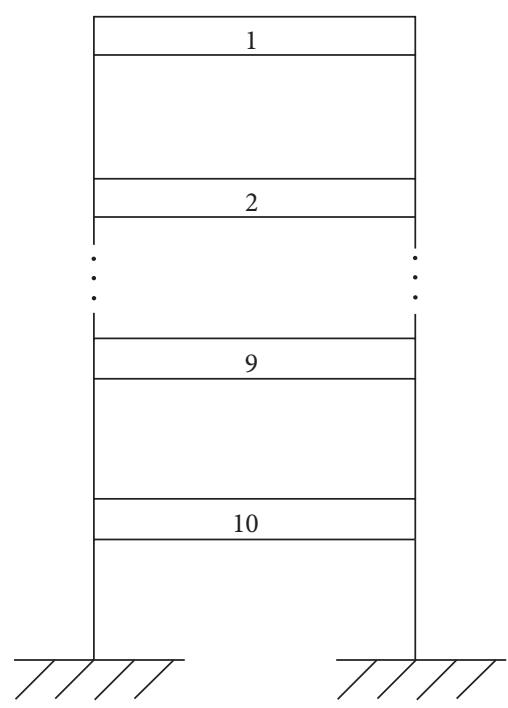

Figure 1: A ten-storey shear frame structure.

$$
\begin{aligned}
& {\left[\begin{array}{cccc}
m_{1} & & & \\
& m_{2} & & \\
& & \vdots & \\
& & & m_{10}
\end{array}\right]\left\{\begin{array}{c}
\ddot{y}_{1} \\
\ddot{y}_{2} \\
\vdots \\
\ddot{y}_{10}
\end{array}\right\}} \\
& +\left[\begin{array}{cccc}
c_{1} & -c_{1} & & \\
-c_{1} & c_{1}+c_{2} & & \\
& & \vdots & c_{9} \\
& & -c_{9} & c_{9}+c_{10}
\end{array}\right]\left\{\begin{array}{c}
\dot{y}_{1} \\
\dot{y}_{2} \\
\vdots \\
\dot{y}_{10}
\end{array}\right\} \\
& +\left[\begin{array}{cccc}
k_{1} & -k_{1} & & \\
-k_{1} & k_{1}+k_{2} & & \\
& & \vdots & -k_{9} \\
& & -k_{9} & k_{9}+k_{10}
\end{array}\right]\left\{\begin{array}{c}
y_{1} \\
y_{2} \\
\vdots \\
y_{10}
\end{array}\right\} \\
& +\left[\begin{array}{cccc}
\alpha_{1} k_{1} & & & \\
-\alpha_{1} k_{1} & \alpha_{2} k_{2} & & \\
& & \vdots & \\
& & -\alpha_{9} k_{9} & \alpha_{10} k_{10}
\end{array}\right]\left\{\begin{array}{c}
\left(y_{1}-y_{2}\right)^{3} \\
\left(y_{2}-y_{3}\right)^{3} \\
\vdots \\
y_{10}^{3}
\end{array}\right\}=\left\{\begin{array}{c}
f_{1} \\
f_{2} \\
\vdots \\
f_{10}
\end{array}\right\} \text {, }
\end{aligned}
$$

where $\alpha_{i}(i=1,2, \ldots, 10)$ is the stiffness hardening coefficient.

In this paper, the solution to the structural positive problem is calculated using the Runge-Kutta method [26].

Suppose that the mass $m_{i}=100 \mathrm{~kg}$, the stiffness $k_{i}=$ $4000 \mathrm{~N} / \mathrm{m}$, the damping $c_{i}=60 \mathrm{Ns} / \mathrm{m}$, and the stiffness hardening coefficient $\alpha_{i}=4 \mathrm{~m}^{-2}(i=1,2, \ldots, 10)$. An unknown load $f(t)$ is applied to the top of the structure, and $f(t)=1000 \sin (1.5 \pi t) N$.

Suppose that the initial value of $k_{i}(i=1,2, \ldots, 10)$ is $5000 \mathrm{~N} / \mathrm{m}$, that of $c_{i}(i=1,2, \ldots, 10)$ is $10 \mathrm{Ns} / \mathrm{m}$, that of $\alpha_{i}(i=1,2, \ldots, 10)$ is $1 \mathrm{~m}^{-2}$, and that of each orthogonal expansion coefficient of the load is 1 .

Without loss of generality, a larger constraint interval is adopted for all unknown parameters. The upper bound of 
the stiffness is 1.5 times its true value, and the lower bound is 0.5 times its true value; the upper bounds of the damping and stiffness hardening coefficient are both 3 times their true values, and the lower bounds are 0 .

5.1.1. Study of the Regularization Parameter. For the dynamic load identification problem, the regularization parameter is crucial because it determines the accuracy of the identified load. Because the sensitivity method itself is an iterative algorithm, the regularization parameter not only affects the accuracy but also determines the convergence speed. Therefore, this section examines how the regularization parameter is determined for the sensitivity method.

The Tikhonov regularization method is used, and the regularization parameter is calculated by the GCV criterion. The acceleration of all degrees of freedom is used for the calculation, the convergence condition is $1 e-10$, and $5 \%$ noise is added to $\ddot{y}$ to simulate the measured response by the following equation:

$$
\ddot{\mathbf{y}}_{n}=\ddot{\mathbf{y}}+\text { level } \cdot \operatorname{std}(\ddot{\mathbf{y}}) \cdot \operatorname{rand},
$$

where $\ddot{\mathbf{y}}_{n}$ is the noise-polluted acceleration, level is the noise level, std(.) represents the standard deviation of a vector, and rand represents a Gaussian white noise sequence with a mean value of zero and a standard deviation of one.

The identified results converge after eight iteration steps, and the regularization parameters of all iteration steps are listed in Table 1 . The convergence processes of $k_{1}, c_{1}$ and $\alpha_{1}$ and a comparison of the identified load with the true load are shown in Figure 2.

As shown in Table 1, for the sensitivity method, when calculated using the GCV criterion, the regularization parameter varies greatly and the largest regularization parameter differs from the smallest regularization parameter by approximately 20 orders of magnitude. At the last iteration step, the regularization parameter is so large that the identified results directly converge.

Therefore, this paper attempts to use a small fixed regularization parameter to carry out the calculation and takes $1 e-7$ as the fixed regularization parameter. The convergence processes of $k_{1}, c_{1}$, and $\alpha_{1}$ and a comparison of the identified load with the true load using the fixed regularization parameter are shown in Figure 3. In addition, multiple calculations are performed using the GCV criterion and the fixed regularization parameter, respectively, and the identified errors are calculated through the evaluation method defined in Section 4. Figure 4 compares the identified errors of all stiffness in two cases.

As shown in Figure 4, when a fixed regularization parameter is used for the calculation, the identified errors of all stiffness are obviously smaller than those obtained when the GCV criterion is used. Therefore, it can be concluded that for the sensitivity method, there is no requirement to calculate the regularization parameter in each iteration step.

To further verify the previous conclusion, different fixed regularization parameters are selected, and the identified errors are listed in Table 2. Because an excessive number of structural parameters are identified in this example, to reduce the amount of data, only the average values of the identified errors of all stiffness, damping, and stiffness hardening coefficients are listed.

With different fixed regularization parameters, the identified errors are the same, indicating that for the sensitivity method, the value of the regularization parameter does not seriously affect the identified results.

Regarding convergence speed, when the value of the fixed regularization parameter varies from $1 e-11$ to $1 e-5$, each calculation requires only 14 iteration steps, but when the fixed regularization parameter is $1 e-4$, the average number of iteration steps required increases by a factor of approximately two. When the fixed regularization parameter is $1 e-3$, the average number of iteration steps required increases to 296, illustrating that the fixed regularization parameter should not be very large.

In addition, some references $[27,28]$ have also made studies on the regularization parameter of the sensitivity method, but the structures adopted are all linear. Moreover, some studies $[29,30]$ show that, when the acceleration is used to identify the load of a linear structure, a low-frequency drift may appear in the identified load and a larger regularization parameter can suppress the drift to some extent. In the examples using nonlinear structures in this paper, there is no obvious low-frequency drift. To get a more general conclusion, further studies are needed on the effect of the regularization parameter on the sensitivity method.

5.1.2. Effect of Response Types. In most cases, the displacement or acceleration is used to solve inverse problems in structural dynamics. To the best of the authors' knowledge, no quantitative comparison of the effect of these two types of response in the field of composite identification has been reported. Therefore, this paper also examines this problem.

In this section, the acceleration and displacement of all degrees of freedom are selected, and 5\% noise is added to the response. Multiple calculations are performed to obtain the identified errors, as listed in Table 3.

It is worth noting that when the displacement is used, the identified error of the load is very large at both ends, as shown in Figure 5, but most of the identified load is very close to the true load. The reason is that the orthogonal expansion has poor fitting results at the two ends of a sequence. Therefore, data for $0.5 \mathrm{~s}$ at both ends of the identified load are first cut off; then, the identified error of the load is calculated using the remaining part.

When the acceleration is used, the identified errors are much smaller than those obtained when the displacement is used. The main reason is that the displacement is obtained by integrating the acceleration twice, which causes the displacement to become very smooth but lose much useful information. However, as mentioned earlier, when the acceleration is used to identify the load of a linear structure, a low-frequency drift may appear, which will make the identified results worse. Therefore, further studies are also needed on the effect of response types on the inverse problems in structural dynamics. 
TABLE 1: The regularization parameters of all iteration steps.

\begin{tabular}{lcccccccc}
\hline Iteration step & 1 & 2 & 3 & 4 & 5 & 6 & 7 \\
\hline Regularization parameter & $7.60 e-12$ & $1.06 e-6$ & $1.67 e-7$ & $8.75 e-7$ & $1.90 e-5$ & $1.10 e-3$ & 0.408 & $3.85 e 9$ \\
\hline
\end{tabular}

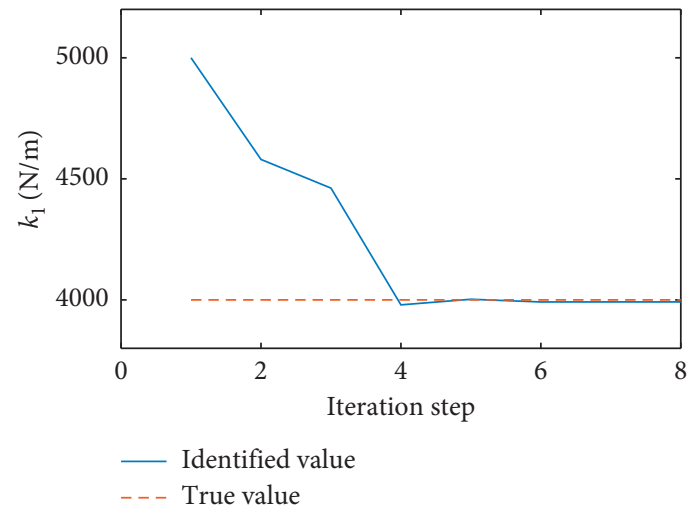

(a)

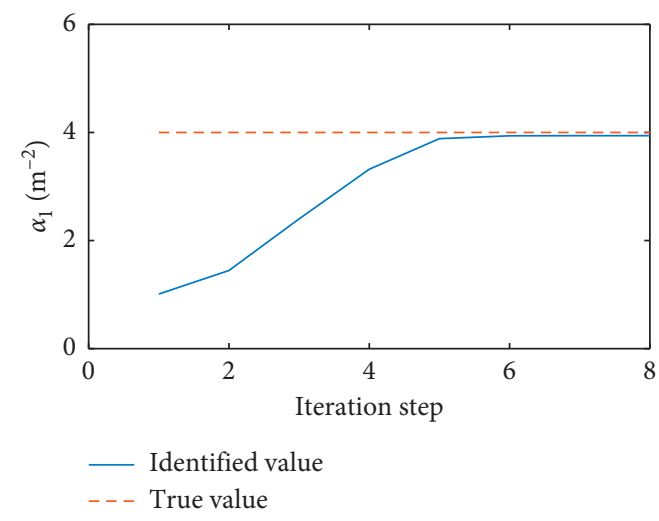

(c)

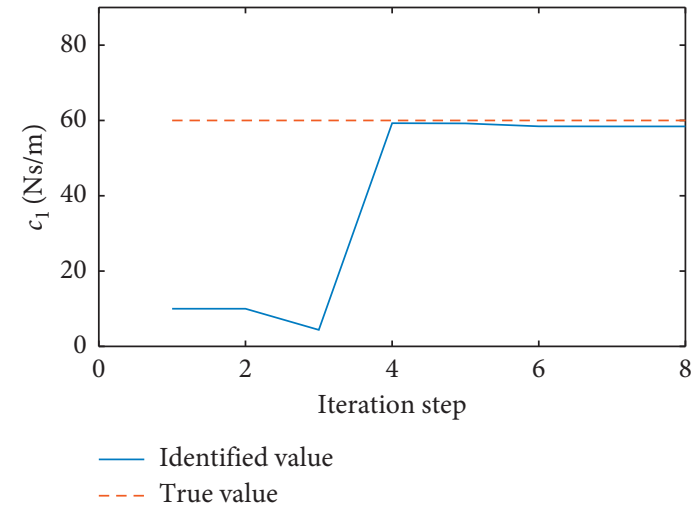

(b)

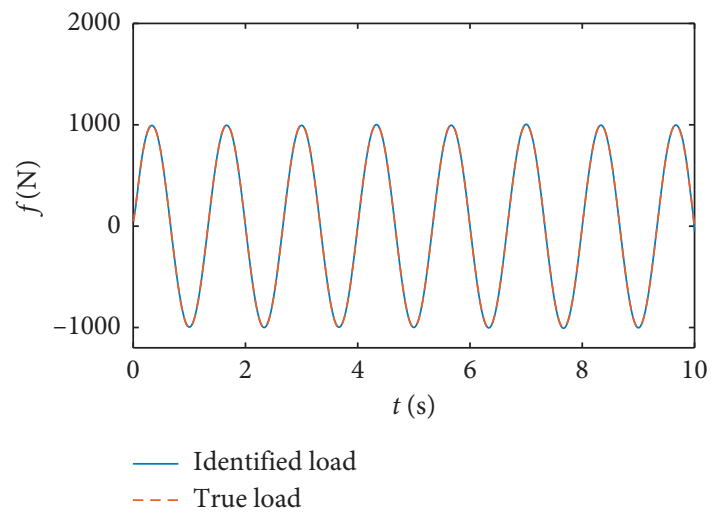

(d)

Figure 2: The convergence processes of (a) $k_{1}$, (b) $c_{1}$, and (c) $\alpha_{1}$ and (d) a comparison of the identified load with the true load using the GCV criterion.

5.1.3. Effect of the Measured Points. To study the effect of the response of each degree of freedom, nine out of ten degrees of freedom are chosen as the measured points, expressed as lack of a certain degree of freedom, and the corresponding identified errors are listed in Table 4.

Moreover, the 2 norms of the acceleration of all degrees of freedom are calculated, and the ratios of the 2 norms of the acceleration of all degrees of freedom to that of degree of freedom 1 are listed in Table 5.

Table 5 shows that the responses of degrees of freedom 1 , $2,6,7$, and 10 are relatively small, illustrating that degrees of freedom with smaller response selected as measured points have a greater effect on the identified results than do those with larger response. The main reason is that, when the measured points are not placed at degrees of freedom with small response, the identified errors of structural parameters at these degrees of freedom will increase a lot, which also leads to a corresponding increase in the average error. However, for a practical engineering structure, the smaller the response, the smaller the signal-to-noise ratio (SNR) of the response, which is also a problem worth considering.
5.1.4. Effects of Noise Levels. This section examines the effect of noise levels on the identified results. Based on the conclusion drawn in the previous section, degrees of freedom 1 , 2, 6, 7, and 10 are selected as the measured points. The identified errors are listed in Table 6.

In the case of five measured points, the identified errors increase with the noise level, but the identified errors are all very small. Even at a high noise level of $20 \%$, the identified error of the damping is less than $6.5 \%$ and that of the stiffness is only approximately $1.2 \%$, illustrating that the sensitivity method is highly accurate.

5.1.5. Effect of the Magnitude of the Perturbation. As mentioned in Section 3.1, the difference method is used to calculate the sensitivity of the structural response with respect to unknown parameters and orthogonal expansion coefficients of loads. Because each unknown quantity has a different effect on the structure, the magnitude of the perturbation is also different to ensure that the sensitivity of the response with respect to each unknown quantity is in the same order of magnitude. 


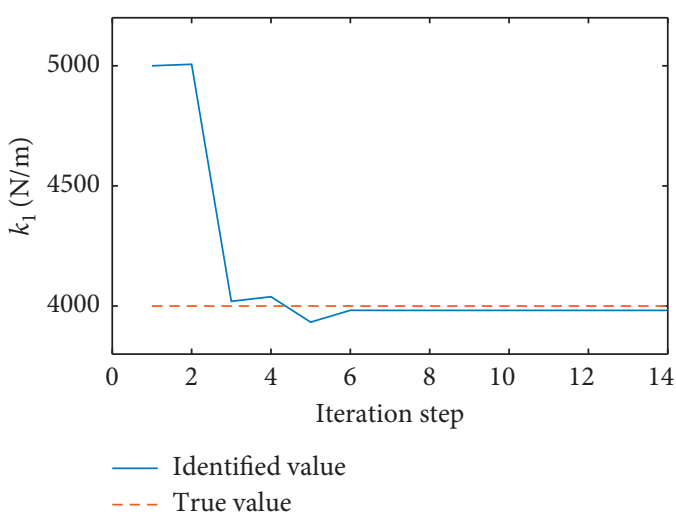

(a)

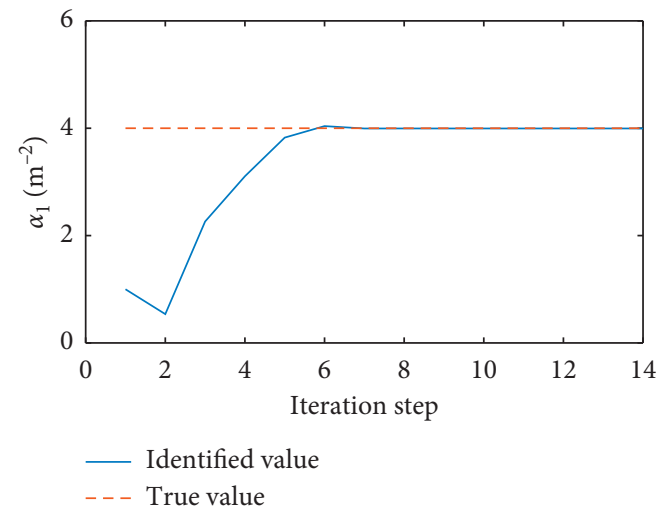

(c)

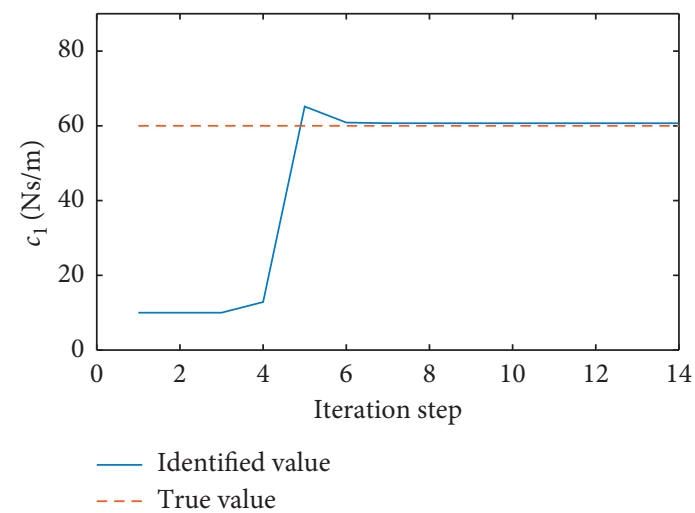

(b)

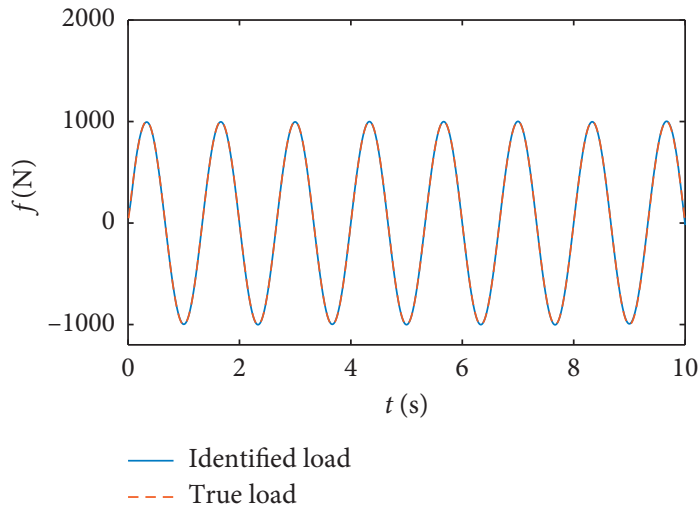

(d)

Figure 3: The convergence processes of (a) $k_{1}$, (b) $c_{1}$, and (c) $\alpha_{1}$ and (d) a comparison of the identified load with the true load using a fixed regularization parameter.

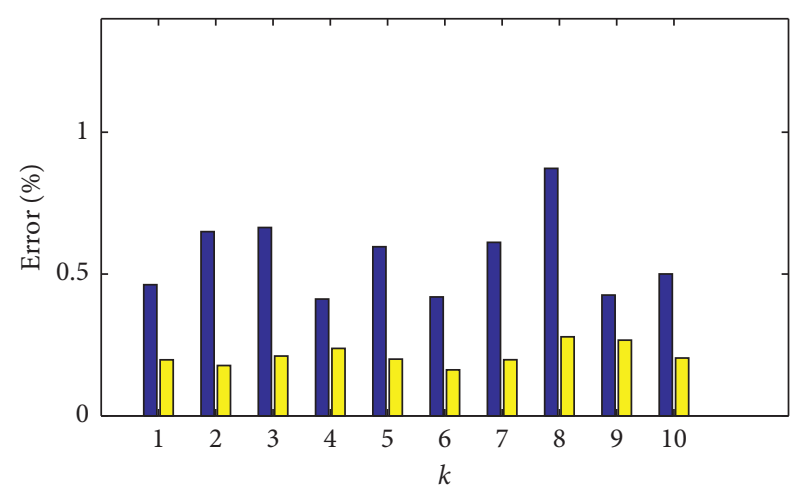

GCV criterion

Fixed regularization parameter

FIgURE 4: A comparison of identified errors of all stiffness between the GCV criterion and a fixed regularization parameter.

The magnitude of the perturbation is defined by the ratio of the perturbation value of an unknown quantity to its true value. With different magnitudes of the perturbation of the stiffness, the identified errors are listed in Table 7, and the magnitudes of the perturbation of other unknown quantities are adjusted accordingly. The noise level is $5 \%$, and other parameters are the same as those in Section 5.1.4.
TABLE 2: Identified errors and average number of iteration steps with different fixed regularization parameters.

\begin{tabular}{lccccc}
\hline $\begin{array}{l}\text { Fixed } \\
\text { regularization } \\
\text { parameter }\end{array}$ & $k(\%)$ & $c(\%)$ & $\alpha(\%)$ & $f(\%)$ & $\begin{array}{c}\text { Average } \\
\text { number of } \\
\text { iteration steps }\end{array}$ \\
\hline $1 e-11$ & 0.213 & 1.417 & 0.843 & 0.625 & 14 \\
$1 e-9$ & 0.213 & 1.417 & 0.843 & 0.625 & 14 \\
$1 e-7$ & 0.213 & 1.417 & 0.843 & 0.625 & 14 \\
$1 e-5$ & 0.213 & 1.417 & 0.843 & 0.625 & 14 \\
$1 e-4$ & 0.213 & 1.417 & 0.843 & 0.625 & 43 \\
$1 e-3$ & 0.213 & 1.417 & 0.843 & 0.625 & 296 \\
\hline
\end{tabular}

As shown in Table 7, the identified errors are basically the same with different magnitudes of the perturbation of the stiffness, illustrating that the magnitude of the perturbation has a little effect on the identified results. Moreover, it is not that the smaller the magnitude of the perturbation, the smaller the identified errors.

5.2. A Bouc-Wen Model Structure. This section further examines the applicability and accuracy of the sensitivity method. For a Bouc-Wen model structure, the vibration equation can be expressed as

$$
\mathbf{M} \ddot{\mathbf{y}}(t)+\mathbf{C} \dot{\mathbf{y}}(t)+\mathbf{K z}(t)=\mathbf{B}_{f} \mathbf{f}(t),
$$




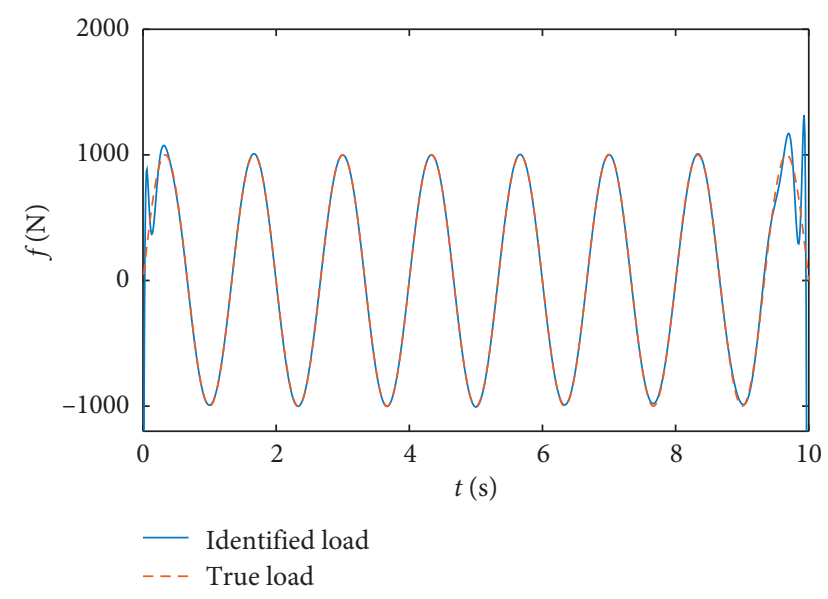

FIgUre 5: A comparison of the identified load with the true load using the displacement.

TABLE 3: Identified errors using the acceleration and displacement.

\begin{tabular}{lcccc}
\hline Response type & $k(\%)$ & $c(\%)$ & $\alpha(\%)$ & $f(\%)$ \\
\hline Acceleration & 0.213 & 1.417 & 0.843 & 0.625 \\
Displacement & 0.653 & 4.611 & 3.682 & 1.592 \\
\hline
\end{tabular}

TABLE 4: Identified errors with different sets of nine measured points.

\begin{tabular}{lllll}
\hline Lack of degree of freedom & $k(\%)$ & $c(\%)$ & $\alpha(\%)$ & $f(\%)$ \\
\hline 1 & 0.244 & 1.556 & 0.902 & 9.607 \\
2 & 0.261 & 1.612 & 0.963 & 0.760 \\
3 & 0.234 & 1.544 & 0.911 & 0.681 \\
4 & 0.221 & 1.439 & 0.856 & 0.640 \\
5 & 0.222 & 1.465 & 0.880 & 0.637 \\
6 & 0.240 & 1.581 & 0.922 & 0.646 \\
7 & 0.237 & 1.583 & 0.916 & 0.648 \\
8 & 0.230 & 1.486 & 0.891 & 0.637 \\
9 & 0.224 & 1.511 & 0.863 & 0.627 \\
10 & 0.253 & 1.557 & 1.076 & 0.641 \\
\hline
\end{tabular}

where $\mathbf{C}$ and $\mathbf{K}$ are the damping and stiffness matrices of the structure, respectively; $\mathbf{z}(t)=\left[\begin{array}{llll}z_{1}(t) & z_{2}(t) & \cdots & z_{N}(t)\end{array}\right]^{\prime}$, which is the hysteresis displacement vector; and $N$ is the number of degrees of freedom. $\dot{z}_{i}(t)$ is expressed as

$$
\begin{array}{r}
\dot{z}_{i}(t)=\dot{y}_{i}(t)-\beta_{i}\left|\dot{y}_{i}(t)\right|\left|z_{i}(t)\right|^{n_{i}-1} z_{i}(t)-\gamma_{i} \dot{y}_{i}(t)\left|z_{i}(t)\right|^{n_{i}}, \\
(i=1,2, \ldots, N),
\end{array}
$$

where $\beta_{i}, \gamma_{i}$, and $n_{i}(i=1,2, \ldots, 10)$ are Bouc-Wen model parameters.

In this section, a ten-storey shear frame structure is selected as the calculation model, as shown in Figure 1. Suppose that $m_{i}=100 \mathrm{~kg}, k_{i}=4000 \mathrm{~N} / \mathrm{m}, c_{i}=60 \mathrm{Ns} / \mathrm{m}$, $\beta_{i}=2, \gamma_{i}=4$, and $n_{i}=2(i=1,2, \ldots, 10)$. An unknown load $f(t)$ is applied to the top of the structure, and $f(t)=1000 \sin (1.5 \pi t) \mathrm{N}$.

Suppose that the initial value of $k_{i}(i=1,2, \ldots, 10)$ is $5000 \mathrm{~N} / \mathrm{m}$, that of $c_{i}(i=1,2, \ldots, 10)$ is $10 \mathrm{Ns} / \mathrm{m}$, that of $\beta_{i}(i=1,2, \ldots, 10)$ is 1 , that of $\gamma_{i}(i=1,2, \ldots, 10)$ is 1 , that of $n_{i}=(i=1,2, \ldots, 10)$ is 1.1 , and that of each orthogonal expansion coefficient of the load is 1 . Unless stated otherwise, $5 \%$ noise is added to the acceleration in the following calculations.

The upper bound of the stiffness is 1.5 times its true value, and the lower bound is 0.5 times its true value; the upper bounds of $c_{i}, \beta_{i}$ and $\gamma_{i}(i=1,2, \ldots, 10)$ are 3 times their true values, and the lower bounds are all 0 ; and the upper bound of $n_{i}=(i=1,2, \ldots, 10)$ is 3 times its true value, and the lower bound is 1 .

5.2.1. Selection of the Regularization Parameter. The study outlined in Section 5.1.1 illustrates that, for a hard-spring model structure, the identified results can always converge within a very large range of the fixed regularization parameter. However, because a Bouc-Wen model structure contains a large number of nonlinear parameters, not only does each iteration step require more time to complete but the convergence processes of the unknown quantities are also much slower. In addition, although the previously mentioned constraint method can play a certain role, the phenomenon of nonconvergence frequently arises. The main reason is that the identified values of structural parameters and loads vary greatly within initial few iteration steps, and in many cases, the response cannot be calculated, resulting in the termination of iteration. Therefore, limiting the magnitude of changes in the identified values of the structural parameters and loads within initial few iteration steps is the fundamental method for solving the problem of nonconvergence.

Therefore, this paper proposes the following method: within initial few iteration steps, a larger regularization parameter is selected; and when the identified values of unknown quantities become relatively stable, the regularization parameter is reduced to ensure that the iteration process converges as quickly as possible.

Based on the method proposed above, the unknown parameters and the load are identified using the acceleration of all degrees of freedom and the convergence condition is $1 e$ - 10. The convergence processes of $k_{1}, c_{1}, \beta_{1}, \gamma_{1}$, and $n_{1}$ and a comparison of the identified load with the true load are shown in Figure 6.

To date, some studies have addressed the composite identification problem for a Bouc-Wen model structure. For example, Lei et al. [31] used the EKF to identify unknown parameters and external loads for a Bouc-Wen model structure, but the parameter $n$ was not identified. $\mathrm{Wu}$ and Smyth [32] compared the identified results between the EKF and unscented Kalman filter (UKF) for a single-degree-offreedom Bouc-Wen model structure under the premise that the load is known. The results show that when EKF is used, the identified errors are very large. Ding et al. [33] used the UKF to identify the unknown parameters and loads of a 3degree-of-freedom Bouc-Wen model structure. For the case in which the response contains no noise, this paper has addressed the example in [33], and the identified errors of unknown parameters are listed in Table 8. It should be noted that the identified results of UKF are obtained from the convergence curves in [33]. 
TABLE 5: The ratios of the 2 norms of the acceleration of all degrees of freedom to that of degree of freedom 1.

\begin{tabular}{lcccccccccc}
\hline Degree of freedom & 1 & 2 & 3 & 4 & 5 & 6 & 7 & 8 & 9 & 10 \\
\hline Ratio & 1.000 & 0.624 & 1.043 & 1.419 & 1.247 & 0.653 & 0.664 & 1.262 & 1.416 & 0.984 \\
\hline
\end{tabular}

TABLE 6: Identified errors with different noise levels.

\begin{tabular}{lllll}
\hline Noise level (\%) & $k(\%)$ & $c(\%)$ & $\alpha(\%)$ & $f(\%)$ \\
\hline 1 & 0.056 & 0.326 & 0.202 & 0.142 \\
5 & 0.272 & 1.701 & 1.027 & 0.730 \\
10 & 0.578 & 3.230 & 1.961 & 1.483 \\
20 & 1.136 & 6.483 & 4.063 & 2.879 \\
\hline
\end{tabular}

TABLE 7: Identified errors with different magnitudes of the perturbation of the stiffness.

\begin{tabular}{|c|c|c|c|c|}
\hline Magnitude of the perturbation (\%) & $k(\%)$ & $c(\%)$ & $\alpha(\%)$ & $f(\%)$ \\
\hline 0.01 & 0.272 & 1.701 & 1.027 & 0.730 \\
\hline 0.1 & 0.272 & 1.701 & 1.027 & 0.730 \\
\hline 1 & 0.272 & 1.701 & 1.026 & 0.729 \\
\hline 10 & 0.280 & 1.740 & 1.048 & 0.736 \\
\hline
\end{tabular}

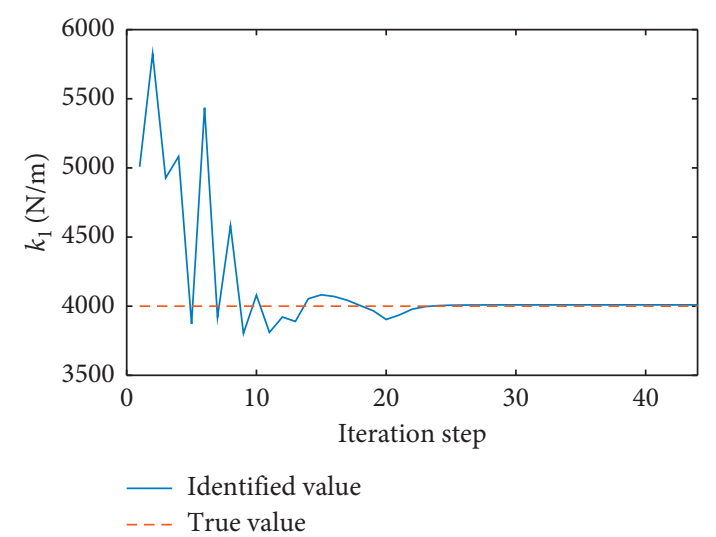

(a)

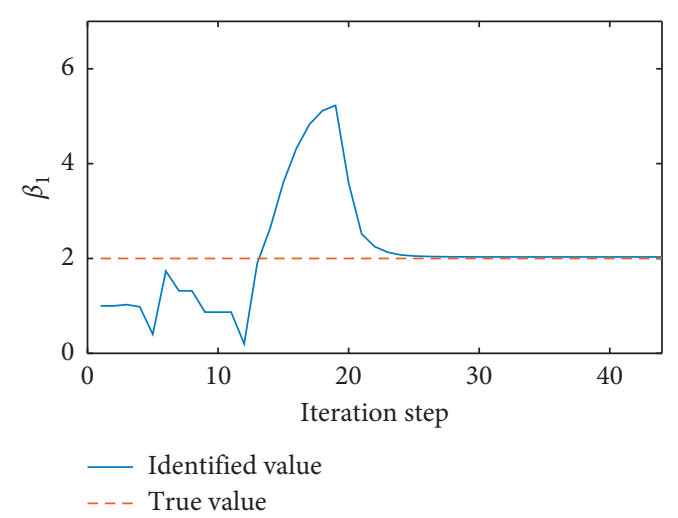

(c)

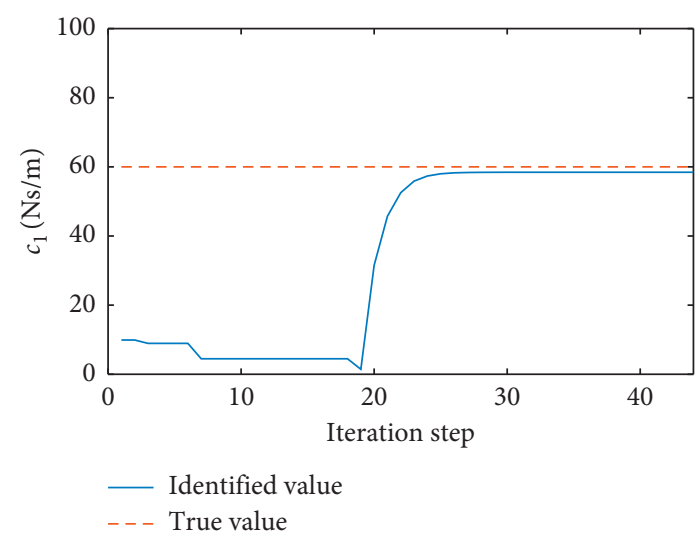

(b)

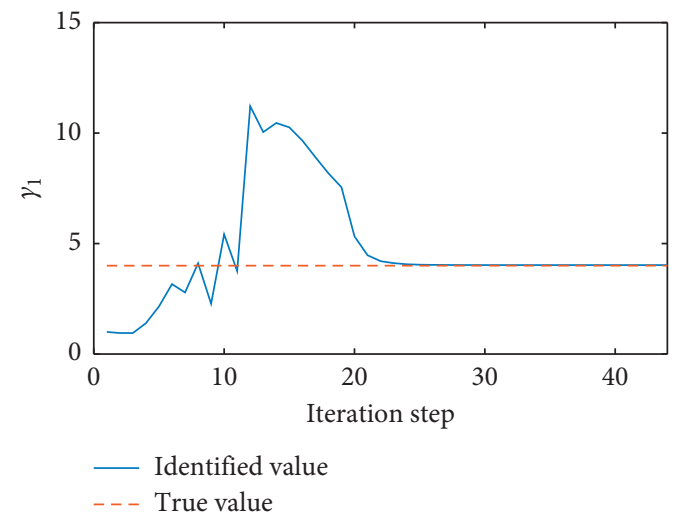

(d)

Figure 6: Continued. 


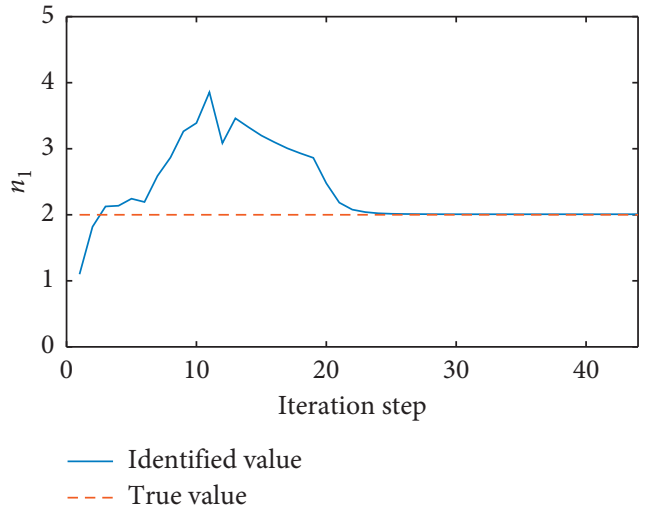

(e)

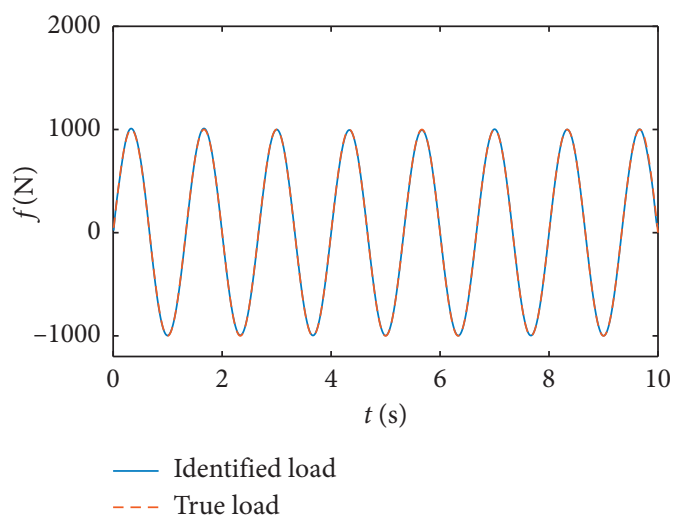

(f)

Figure 6: The convergence processes of (a) $k_{1}$, (b) $c_{1}$, (c) $\beta_{1}$, (d) $\gamma_{1}$, and (e) $n_{1}$ and (f) a comparison of the identified load with the true load.

TABLE 8: Identified errors using the sensitivity method and UKF [33].

\begin{tabular}{lcccccccccc}
\hline \multirow{2}{*}{ Degree of freedom } & \multicolumn{9}{c}{ Sensitivity method } \\
& $k(\%)$ & $c(\%)$ & $\beta(\%)$ & $\gamma(\%)$ & $n(\%)$ & $k(\%)$ & $c(\%)$ & $\beta(\%)$ & $\gamma(\%)$ & $n(\%)$ \\
\hline 1 & 0 & 0.02 & 0.07 & 0.07 & 0.07 & 0.6 & 1.4 & 6.9 & 0.8 & 2.9 \\
2 & 0.01 & 0.08 & 0.15 & 0.17 & 0.06 & 0.3 & 1.3 & 3.9 & 0 & 1.6 \\
3 & 0.05 & 0.01 & 0.21 & 0.02 & 0.06 & 0.5 & 0 & 5.9 & 3.1 & 2.9 \\
\hline
\end{tabular}

TABLE 9: The ratios of the 2 norms of the acceleration of all degrees of freedom to that of degree of freedom 1.

\begin{tabular}{lcccccccccc}
\hline Degree of freedom & 1 & 2 & 3 & 4 & 5 & 6 & 7 & 8 & 9 & 10 \\
\hline Ratio & 1.000 & 0.874 & 0.654 & 0.748 & 0.928 & 0.705 & 0.365 & 0.624 & 0.831 & 0.623 \\
\hline
\end{tabular}

It can be observed that even if the noise is not considered, the identified errors using the UKF are large, but the sensitivity method exhibits good accuracy. In addition, the convergence processes of the unknown parameters in [33] fluctuate so much that it is difficult to judge whether the identified values converge.

5.2.2. Effect of the Distribution of Measured Points. Based on the conclusion drawn in Section 5.1.3, the ratios of the 2 norms of the acceleration of all degrees of freedom to that of degree of freedom 1 are listed in Table 9. It can be observed that the acceleration of degrees of freedom $3,7,8$, and 10 is relatively small.

In addition, several sets of five measured points with better identified results and the corresponding identified errors are listed in Table 10, arranged in descending order of the identified errors.

Many sets in Table 10 contain degrees of freedom 1, 3, 7, and 10, illustrating that the acceleration of these degrees of freedom has a strong effect on the identified results, which is consistent with the conclusion drawn previously. However, the identified error of Set 5 is not the smallest, and the identified error of Set 2 is relatively large. The reason is that the distribution of measured points in Sets 2 and 5 is more concentrated such that the information contained in the response involves greater repetition. When the measured
TABLE 10: Identified errors of several sets of five measured points.

\begin{tabular}{cccccccc}
\hline Set & $\begin{array}{c}\text { Degrees of } \\
\text { freedom }\end{array}$ & $k(\%)$ & $c(\%)$ & $\beta(\%)$ & $\gamma(\%)$ & $n(\%)$ & $f(\%)$ \\
\hline 1 & $1,3,6,8,10$ & 0.402 & 2.721 & 6.807 & 6.170 & 2.395 & 1.057 \\
2 & $1,2,3,7,10$ & 0.417 & 2.966 & 6.752 & 5.982 & 2.290 & 0.868 \\
3 & $1,2,5,7,10$ & 0.421 & 2.916 & 6.821 & 5.758 & 2.240 & 0.985 \\
4 & $1,2,4,7,10$ & 0.383 & 2.772 & 6.487 & 5.701 & 2.249 & 0.901 \\
5 & $1,3,7,8,10$ & 0.418 & 2.708 & 6.249 & 5.853 & 2.274 & 1.070 \\
6 & $1,3,4,7,10$ & 0.360 & 2.530 & 6.156 & 5.585 & 2.194 & 0.953 \\
7 & $1,3,5,7,10$ & 0.395 & 2.719 & 6.104 & 5.358 & 2.074 & 1.042 \\
8 & $1,3,6,7,10$ & 0.383 & 2.583 & 6.100 & 5.434 & 2.116 & 1.032 \\
\hline
\end{tabular}

points are relatively dispersed, the identified results will not be excessively unfavourable, such as in Sets 1, 3, and 4 .

In fact, the identified errors of all sets in Table 10 do not vary greatly. In addition, considering that this example uses five measured points to identify fifty unknown parameters and an unknown load, the identified errors listed in Table 10 and some unlisted results are completely acceptable, illustrating that the sensitivity method has extremely high accuracy.

\section{Conclusion}

Because the governing equation of the sensitivity method does not depend on the structural vibration equation, when 
the sensitivity method is applied to a more complex problem, the establishment of and the solution to the governing equation do not become very difficult, which represents the greatest advantage of this method. In this paper, the key issues associated with the sensitivity method are systematically discussed, and suggestions for improvement are proposed and verified by two nonlinear model examples. Moreover, the discussion of the response types, distribution of measured points, and the magnitude of the perturbation can provide some guidance for engineering applications.

\section{Data Availability}

All data used to support the findings of this study are included within the article.

\section{Conflicts of Interest}

The authors declare that there are no conflicts of interest regarding the publication of this paper.

\section{Acknowledgments}

The authors gratefully acknowledge support for this research from the National Natural Science Foundation of China (Grant No. 51579084), Jiangsu Province Key R\&D Project (Grant No. BE2017167), and the Fundamental Research Funds for the Central Universities (Grant Nos. 2018B48514 and 2018B685X14).

\section{References}

[1] C. R. Farrar and K. Worden, "An introduction to structural health monitoring," in New Trends in Vibration Based Structural Health Monitoring, A. Deraemaeker and K. Worden, Eds., pp. 1-17, Springer Vienna, Vienna, Austria, 2010.

[2] K. Y. Wong, "Design of a structural health monitoring system for long-span bridges," Structure and Infrastructure Engineering, vol. 3, no. 2, pp. 169-185, 2007.

[3] W. Fan and P. Qiao, "Vibration-based damage identification methods: a review and comparative study," Structural Health Monitoring, vol. 10, no. 1, pp. 83-111, 2010.

[4] J. Sanchez and H. Benaroya, "Review of force reconstruction techniques," Journal of Sound and Vibration, vol. 333, no. 14, pp. 2999-3018, 2014.

[5] C. G. Koh, B. Hong, and C. Y. Liaw, "Parameter identification of large structural systems in time domain," Journal of Structural Engineering, vol. 126, no. 8, pp. 957-963, 2000.

[6] S. R. Ibrahim, "Random decrement technique for modal identification of structures," Journal of Spacecraft and Rockets, vol. 14 , no. 11 , pp. 696-700, 1977.

[7] K. Toki, T. Sato, and J. Kiyono, "Identification of structural parameters and input ground motion from response time histories," Doboku Gakkai Ronbunshu, vol. 6, no. 410, pp. 243-251, 1989.

[8] E. Reynders, "System identification methods for (operational) modal analysis: review and comparison," Archives of Computational Methods in Engineering, vol. 19, no. 1, pp. 51-124, 2012.

[9] H. Akaike, "Power spectrum estimation through autoregressive model fitting," Annals of the Institute of Statistical Mathematics, vol. 21, no. 1, pp. 407-419, 1969.
[10] J.-N. Juang, J. E. Cooper, and J. R. Wright, “An eigensystem realization algorithm using data correlations (ERA/DC) for modal parameter identification," Control Theory and Advanced Technology, vol. 4, pp. 5-14, 1988.

[11] E. Mrabet, M. Abdelghani, and N. Ben Kahla, "A new criterion for the stabilization diagram used with stochastic subspace identification methods: an application to an aircraft skeleton," Shock and Vibration, vol. 2014, Article ID 409298, 8 pages, 2014.

[12] O. Maruyama, E. Saito, and M. Hoshiya, "Identification of dynamic parameters of a running load and beam system," Doboku Gakkai Ronbunshu, vol. 113, no. 368, pp. 283-292, 1986.

[13] M. Hoshiya and O. Maruyama, "Identification of running load and beam system," Journal of Engineering Mechanics, vol. 113, no. 6, pp. 813-824, 1987.

[14] J. N. Yang, S. W. Pan, and S. L. Lin, "Least-squares estimation with unknown excitations for damage identification of structures," Journal of Engineering Mechanics, vol. 133, no. 1, pp. 12-21, 2007.

[15] J. N. Yang and H. Huang, "Sequential non-linear least-square estimation for damage identification of structures with unknown inputs and unknown outputs," International Journal of Non-Linear Mechanics, vol. 42, no. 5, pp. 789-801, 2007.

[16] T. F. Mu, L. Zhou, and J. N. Yang, "Experimental study of an adaptive sequential nonlinear LSE with unknown inputs for structural damage tracking," Shock and Vibration, vol. 2014, Article ID 294163, 14 pages, 2014.

[17] X. Q. Zhu and S. S. Law, "Damage detection in simply supported concrete bridge structure under moving vehicular loads," Journal of Vibration and Acoustics, vol. 129, no. 1, pp. 58-65, 2006.

[18] Z. R. Lu and S. S. Law, "Identification of system parameters and input force from output only," Mechanical Systems and Signal Processing, vol. 21, no. 5, pp. 2099-2111, 2007.

[19] Q. Zhang, Ł. Jankowski, and Z. Duan, "Identification of coexistent load and damage," Structural and Multidisciplinary Optimization, vol. 41, no. 2, pp. 243-253, 2010.

[20] Q. Zhang, Ł. Jankowski, and Z. Duan, "Simultaneous identification of moving masses and structural damage," Structural and Multidisciplinary Optimization, vol. 42, no. 6, pp. 907-922, 2010.

[21] B. Xu, J. He, R. Rovekamp, and S. J. Dyke, "Structural parameters and dynamic loading identification from incomplete measurements: approach and validation," Mechanical Systems and Signal Processing, vol. 28, pp. 244-257, 2012.

[22] A. N. Tikhonov, "On the solution of incorrectly stated problems and a method of regularization," Doklady Akademii Nauk, vol. 151, pp. 501-504, 1963.

[23] P. C. Hansen, "Analysis of discrete ill-posed problems by means of the L-curve," Siam Review, vol. 34, no. 4, pp. 561580, 1992.

[24] G. H. Golub, M. Heath, and G. Wahba, "Generalized crossvalidation as a method for choosing a good ridge parameter," Technometrics, vol. 21, no. 2, pp. 215-223, 1979.

[25] R. D. Fierro and P. C. Hansen, "Accuracy of TSVD solutions computed from rank-revealing decompositions," Numerische Mathematik, vol. 70, no. 4, pp. 453-471, 1995.

[26] R. Cortell, "Application of the fourth-order Runge-Kutta method for the solution of high-order general initial value problems," Computers \& Structures, vol. 49, no. 5, pp. 897900, 1993.

[27] K. Zhang, Study on Simultaneous Identification of Structural Damage and Load Based on Incomplete Measurements, Harbin Institute of Technology, Harbin, China, 2010, in Chinese. 
[28] K. Zhang and X. H. Guo, "Adaptive Tikhonov regularization for simultaneous identification of structural parameters and input," in Proceedings of International Conference on Material Science and Civil Engineering (Msce 2016), pp. 494-500, Guilin, China, August 2016.

[29] S. E. Azam, E. Chatzi, and C. Papadimitriou, "A dual Kalman filter approach for state estimation via output-only acceleration measurements," Mechanical Systems and Signal Processing, vol. 60-61, pp. 866-886, 2015.

[30] F. Naets, J. Cuadrado, and W. Desmet, "Stable force identification in structural dynamics using Kalman filtering and dummy-measurements," Mechanical Systems and Signal Processing, vol. 50-51, pp. 235-248, 2015.

[31] Y. Lei, Y. Wu, and T. Li, "Identification of non-linear structural parameters under limited input and output measurements," International Journal of Non-Linear Mechanics, vol. 47, no. 10, pp. 1141-1146, 2012.

[32] M. Wu and A. W. Smyth, "Application of the unscented Kalman filter for real-time nonlinear structural system identification," Structural Control and Health Monitoring, vol. 14, no. 7, pp. 971-990, 2007.

[33] Y. Ding, L. N. Guo, and B. Y. Zhao, "Parameter identification for nonlinear structures by a constrained Kalman filter with limited input information," International Journal of Structural Stability and Dynamics, vol. 17, no. 1, article 1750010, 2016. 


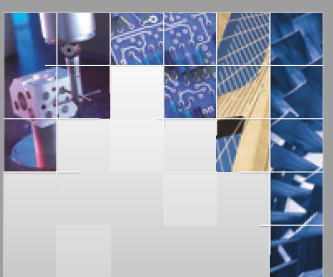

\section{Enfincering}
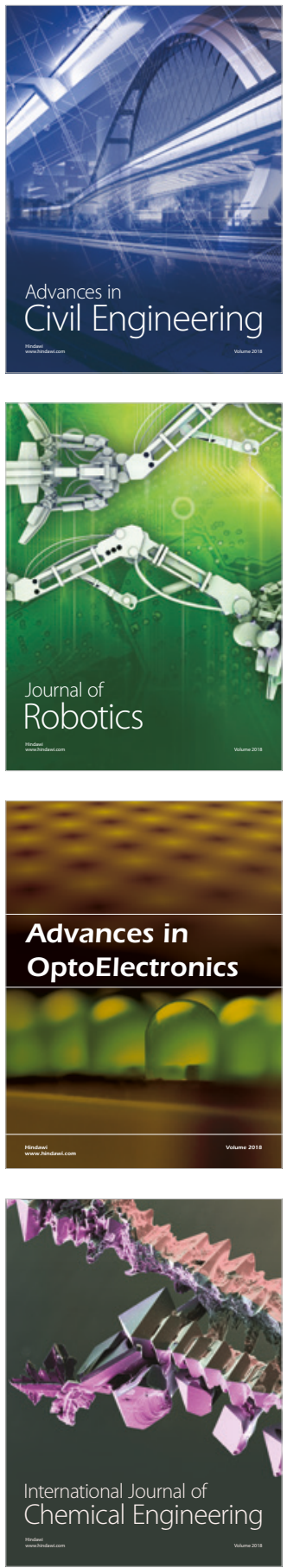

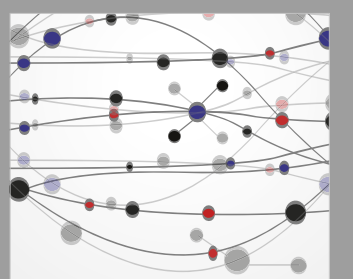

\section{Rotating \\ Machinery}

The Scientific World Journal

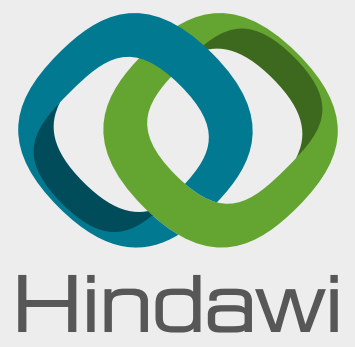

Submit your manuscripts at

www.hindawi.com
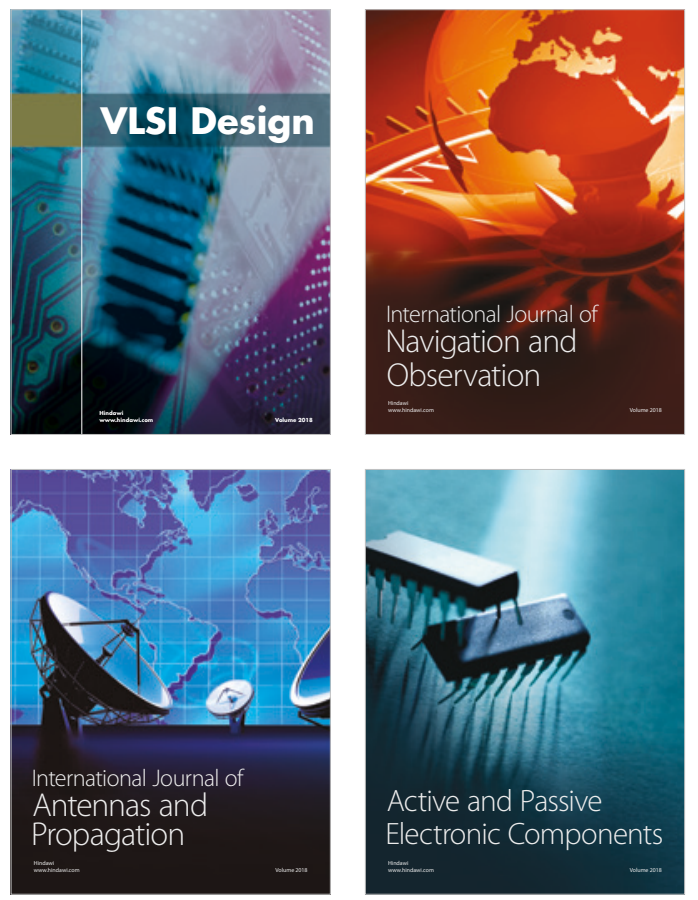
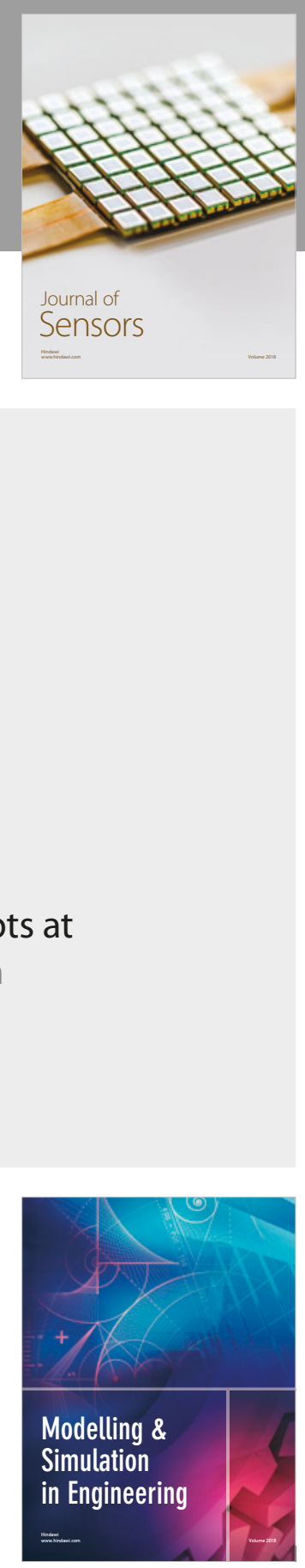

\section{Advances \\ Multimedia}
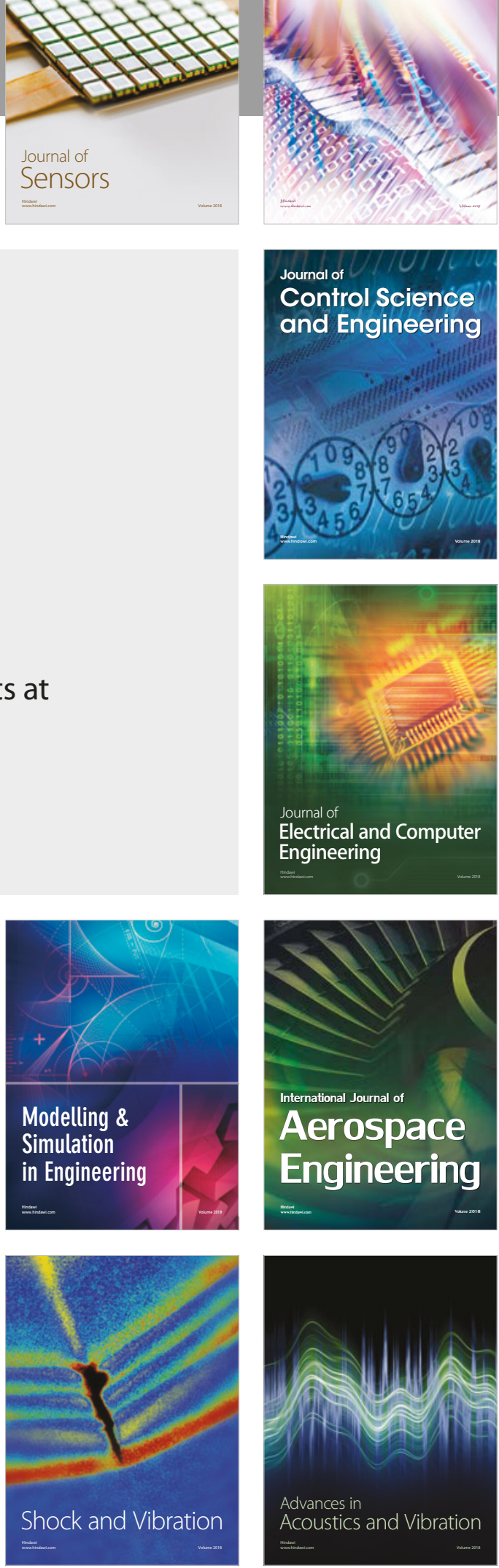